\title{
Arbitrary stability for a Hopfield neural network problem with discrete and distributed delays
}

\author{
Mohammed Kassim ${ }^{1}$ and Nasser-eddine Tatar ${ }^{2}$ \\ ${ }^{1}$ Imam Abdulrahman Bin Faisal University \\ ${ }^{2}$ King Fahd University of Petroleum and Minerals
}

July 8, 2021

\begin{abstract}
We consider a Hopfield neural network system containing discrete as well as distributed delays. A stability result of arbitrary type is proved under weaker assumptions than the used ones so far. This result includes exponential and polynomial (or power type) stability as special cases. Our proof relies on a judicious choice of Lyapunov-type functionals and some appropriate manipulations.
\end{abstract}

\section{Hosted file}

manuscript.pdf available at https://authorea.com/users/398483/articles/529535-arbitrarystability-for-a-hopfield-neural-network-problem-with-discrete-and-distributed-delays 